\title{
BMJ Open Perceptions of ASHA workers in the HOPE collaborative care mental health intervention in rural South India: a qualitative analysis
}

\author{
Stuti Bansal (D) , ${ }^{1}$ Krishnamachari Srinivasan, ${ }^{2}$ Maria Ekstrand ${ }^{2,3}$
}

To cite: Bansal S, Srinivasan K, Ekstrand M. Perceptions of ASHA workers in the HOPE collaborative care mental health intervention in rural South India: a qualitative analysis. BMJ Open 2021;11:e047365. doi:10.1136/ bmjopen-2020-047365

- Prepublication history and additional supplemental material for this paper are available online. To view these files, please visit the journal online (http://dx.doi.org/10.1136/ bmjopen-2020-047365).

Received 25 November 2020 Accepted 20 September 2021

Check for updates

C Author(s) (or their employer(s)) 2021. Re-use permitted under CC BY-NC. No commercial re-use. See rights and permissions. Published by BMJ.

${ }^{1}$ Department of Molecular and Cellular Biology, University of California Berkeley, Berkeley, California, USA

${ }^{2}$ Division of Mental Health \& Neurosciences, St John's Research Institute, Bangalore, Karnataka, India

${ }^{3}$ Division of Prevention Science, Center for AIDS Prevention

Studies, University of California San Francisco, San Francisco, California, USA

Correspondence to

Stuti Bansal;

sbansal@berkeley.edu

\section{ABSTRACT}

Objective The main objective of this exploratory study was to investigate the overlooked perspectives and beliefs of Accredited Social Health Activists (ASHA workers) regarding a collaborative care mental health intervention (HOPE: Healthier Options through Empowerment), mental illness and the health of their rural communities.

Design Semi-structured, one-on-one, qualitative interviews.

Setting Seven primary health centres (PHCs) in rural Karnataka, India. All PHCs had previously completed the HOPE study.

Participants 15 ASHA workers, selected via purposive sampling. ASHAs are high schooleducated village women trained as community health workers. ASHAs were included if they had previously participated in the HOPE intervention, a collaborativecare randomised controlled trial that aimed to integrate mental healthcare into existing primary care systems in rural Karnataka.

Interventions No interventions were introduced.

Results ASHA workers mostly had positive interactions with patients, including encouraging them to attend sessions, helping to explain the topics and techniques, and checking on the patients frequently. ASHA workers were able to identify key barriers to treatment and facilitators to treatment. ASHAs claimed that their knowledge about mental illness improved because of the HOPE study, though gaps remained in their understanding of aetiology and treatment. Several expressed interest in receiving additional mental health training. Overall, ASHAs viewed the HOPE study as a necessary and effective intervention, and requested that it expand.

Conclusions This paper discusses the perspectives of ASHAs who participated in a novel effort to extend the collaborative care model to their own communities. ASHA workers help maintain relationships with patients that encourage participation, and the efforts of ASHAs often aid in mitigating common barriers to treatment. ASHA workers' beliefs and knowledge regarding mental illness can be changed, and ASHAs can become effective advocates for patients. Future collaborative care interventions would likely benefit from involving ASHA workers in community outreach efforts.
Strengths and limitations of this study

- One-on-one qualitative interviews allowed for in depth understanding of the Accredited Social Health Activists (ASHA) workers' beliefs and opinions, which have not been adequately researched.

- ' This study focuses on the ASHA workers' own perspectives regarding the HOPE study (Healthier Options through Empowerment), of which they were an integral part. Discussing this with the ASHAs in detail allowed for an understanding of how feasible it will be in the future to extend similar collaborative care interventions to communities by working directly with ASHAs.

- Real-time translation by an interpreter may have obscured some complexity, particularly any cultural nuances.

- Some ASHA workers completed their positions with the HOPE study more than a year prior to their interview, which may have interfered with their recall.

\section{BACKGROUND}

Mental illness, particularly depression, is a leading cause of disability globally ${ }^{12}$ and India accounts for $15 \%$ of the global mental, neurological, and substance use disorder (MNSUD) burden. ${ }^{34}$ Common mental disorders (CMD) make up a large part of the mental illness disease burden, with depression and anxiety together accounting for almost $50 \%$ of MNSUD-caused disability adjusted life years. CMD are often highly comorbid with noncommunicable diseases (NCD) like diabetes and cardiovascular disease,${ }^{56}$ which is doubly concerning as the burden of disease due to NCD continues to rise rapidly across India. ${ }^{7}$

There is a severe lack of mental health resources in India, with roughly one psychiatrist per 300000 people $^{89}$ and in rural areas there is one psychiatrist per 500000 people. ${ }^{10} 11$ According to the 2011 Indian census, $68.8 \%$ of the Indian population live in rural areas, making the lack of mental health resources more challenging. ${ }^{12}$ In rural India, 
healthcare is often dependent on the efforts of Accredited Social Health Activists (ASHA). In 2005, the ASHA programme was established in India as a component of the National Rural Health Mission to address the shortage of healthcare workers in rural areas. ${ }^{13}$ It recruits high school educated village women to be trained as community health workers. There is one ASHA worker per village of approximately 1000 residents. ASHA workers receive training in basic healthcare, and work primarily in the field of maternal and child health. They also participate in health education campaigns on nutrition, sanitation, and basic health. ${ }^{1415}$

ASHA workers have been effectively involved in a variety of interventions targeting conditions like HIV, ${ }^{16-20}$ mental health ${ }^{21}$ and NCD, ${ }^{22}$ among others. However, studies have noted that ASHA workers display stereotyped beliefs about people with mental illness, ${ }^{23}$ and have very little understanding of clinical depression. ${ }^{24}$

Perspectives of ASHA workers in general have been greatly overlooked, and the few studies that investigated them largely focused on the motivations of ASHA workers. ${ }^{25}{ }^{26}$ As more ASHA workers take on additional responsibilities, it is important to understand their experiences and beliefs, particularly about community-based mental health interventions. The aim of this qualitative study was to explore the perspectives and beliefs of the ASHA workers who participated in the HOPE study (Healthier Options through Empowerment), a cluster randomised controlled trial (RCT) which used a collaborative care model to integrate mental health treatment in primary care clinics in a rural district of Karnataka. ${ }^{27}$ In particular, the HOPE RCT used the efforts of ASHA workers to extend the collaborative care model directly to the community, and this study explores the ASHAs' perspectives regarding their experience with HOPE.

\section{METHODS}

This study is a follow-up to the parent HOPE RCT that is implementing and evaluating the integration of mental health interventions in primary care. ${ }^{27}$ The HOPE intervention is a multi-level RCT driven largely by social cognitive theory (SCT), targeting patients with depression or anxiety, comorbid with diabetes or cardiovascular disease. $\mathrm{SCT}^{28}{ }^{29}$ is a good fit for promoting behavioural change linked to cardiovascular and mental health through its emphasis on interpersonal interactions and support, observational learning and improving self-efficacy. The HOPE study is guided by the SCT framework on multiple levels. First, it takes advantage of bidirectional interactions between people and their environments by using community health fairs, training the staff at primary health clinics, using peer groups, obtaining family support and especially through the efforts of the ASHA workers. Second, it uses the SCT construct of 'observational learning' through 'Healthy Living Group' (HLG) sessions, where groups of participants learn skills together and form informal buddy systems. Finally, it encourages behaviour change through skills training and feedback at these HLG sessions, which promotes self-efficacy and self-confidence. ${ }^{27-29}$

HOPE aims to use a collaborative care model to improve the screening, diagnosis, and treatment of CMD and NCD by holding community health screening fairs, training doctors and nurses in mental health diagnosis and treatment, and using ASHA workers to colead the community-based HLG sessions and interact directly with the community. In the HOPE study, the collaborative care model, which is usually limited to the clinic, was extended to the community level, relying heavily on the efforts of ASHA workers. ASHAs performed the role of liaison between patients and primary health centre (PHC) staff, which included conducting home visits and delivering reminders to HOPE patients about appointments. In addition, ASHA workers helped run the health screening fairs and cofacilitated 21 HLG sessions with trained research staff designed to improve patient health behaviours through information, skills-building, feedback and social support. ${ }^{27}$

This analysis used a qualitative grounded-theory approach to understand the perspectives of ASHA workers who participated in HOPE. This approach was used to obtain richer data in the ASHAs' own words. Prior to the study, little was understood about the ASHAs' beliefs, which precluded a quantitative approach.

\section{Patient and public involvement}

Patients or the public were not involved in the design, conduct, reporting or dissemination of this research.

\section{Study participants}

Following pilot testing, study staff recruited 15 HOPE study ASHA workers from seven PHCs where the study had been implemented. ASHA workers were recruited via purposive sampling methods and enrolled following written informed consent. ASHA workers were included only if they worked at a PHC that completed the intervention, and were involved in intervention procedures like screening fairs, HLG sessions and home visits. During recruitment, ASHAs from more recently completed PHCs were prioritised to ensure that the interviewed ASHAs would remember enough to answer questions in depth. Recruitment stopped on thematic saturation of the interviews.

\section{Instruments and procedures}

The ASHA workers, all of whom had recently completed the HOPE intervention, each completed a 45-60 min semi-structured qualitative interview in the summer of 2018. Interviews were administered at their nearest PHC by the study researcher (SB) and an interpreter who was conversant in Kannada and English. The interview guide included questions about the ASHA worker's role during the screening fairs and HLG sessions, her experiences with individual patients, her experiences with home visits, 
and her thoughts on the HOPE study (see online supplemental appendix 1).

$\mathrm{SB}$ is a junior researcher from the USA and therefore relied on study staff and senior researchers for guidance. The study guide and interview procedures were designed with feedback from staff who were involved in HOPE, as they were more aware of cultural context and language differences, and had worked with the ASHA workers. The guide was translated into Kannada and back-translated into English, and discrepancies were resolved. The interview guide was pilot tested with two participants, and the questions and procedures were subsequently finalised with input from the study manager.

Since the ASHA workers spoke only Kannada, SB asked questions in English, and the interpreter translated the questions into Kannada. The responses of the ASHA workers were translated into English. The interpreter worked with many of the ASHAs during the HOPE intervention, and was therefore trustworthy to them. She was also familiar with many of their experiences and able to ask for more details and context where necessary. To minimise social desirability, ASHAs were told that there were no right or wrong answers and that their names and other identifying information would be kept confidential. They were encouraged to discuss their true experiences, and told that their honest feedback would be helpful in designing future programmes.

Interviews were audio recorded with permission from the participants. The English translations of the ASHA interviews were manually transcribed, and the transcriptions were verbatim except for minor grammatical changes for clarity. Each ASHA worker was compensated 150 INR (US\$2.08) for travel expenses.

\section{Analyses}

Fifteen transcripts were coded by using Dedoose, an open coding platform, to apply codes to excerpts and organise coded material within a thematic framework. This framework was devised through line-by-line coding and the grounded theory approach, and was subsequently applied to each interview. The framework underwent editing whenever new themes appeared. Final themes were determined when additional interviews no longer yielded new themes (ie, thematic saturation), and any redundant codes were merged. Excerpts were edited lightly for clarity.

\section{RESULTS}

Table 1 summarises the demographic characteristics of the 15 participants. When asked how many patients they served for HOPE, and how many HLG sessions they attended, several ASHA workers gave ranges, or were unable to come up with numbers. In the case of ranges, the lower number of the range was taken to calculate an approximate mean. The included ASHAs had on average an age of 38.6 years, 10.13 years of education and 8.67 years of experience in the government ASHA programme.
Table 1 Sample characteristics

\begin{tabular}{lrl}
\hline Characteristic & Range & Mean \\
\hline Age & $28-46$ & 38.6 \\
Number of patients served in HOPE study & $5-15$ & $\sim 10$ \\
Years in ASHA programme & $3-11$ & 8.67 \\
Number of HLG sessions attended & $5-21$ & $\sim 12.67$ \\
Years of education & $7-12$ & 10.13 \\
\hline
\end{tabular}

ASHA, Accredited Social Health Activists; HLG, Healthy Living Group; HOPE, Healthier Options through Empowerment.

They each worked with approximately 10 patients for the HOPE study, and attended 12.67 HLG sessions.

Over the course of 15 qualitative interviews, ASHA workers revealed their perspectives on their participation in the HOPE study and their beliefs about mental illness and NCD. The themes that emerged from these interviews were: interactions between ASHA workers and patients, ASHA workers' knowledge and beliefs about mental illness, barriers and facilitators to treatment, and ASHA workers' perspectives on the HOPE study.

\section{ASHA-patient interactions}

The ASHA workers described their interactions with patients as positive and productive, and these interactions were crucial to recruitment and engaging patients. ASHA workers confirmed that they helped to explain the study to patients who needed clarification and motivate patients to come to the health screening fairs, enrol in the study and attend the HLG sessions. They stated that they encouraged patients to follow health advice and practice diet changes, exercise, and stress management techniques like yoga and breathing exercises, both during group sessions and home visits. ASHAs mostly believed that patients listened to them and took their advice.

However, not all interactions with patients were positive. Some ASHA workers mentioned that they were uncomfortable interacting with certain patients because the ASHAs did not have sufficient knowledge about relevant topics.

Before I was not aware of the topic ... and if I tell incomplete knowledge maybe the [patients] won't accept, I was thinking like that. Later I got to know more information about smoking, smokeless tobacco, and all, I am comfortably speaking now. [A104]

Additionally, several ASHA workers mentioned that some of their patients were hostile towards them, which led to the ASHA workers avoiding interactions with these patients-some patients refused to listen to the ASHA workers, insulted them, and told them they knew nothing.

The patients were telling me that they are elder than us, so why should they listen to our words. That we don't know anything, they know everything, like that they were telling. They said that other people will tell me things, and then I am coming and telling these 
things to them-I don't know anything, but they [patients] are experienced and know everything. [A113]

\section{ASHA knowledge and beliefs about mental illness}

Many of the ASHA workers reported that they had held misconceptions about mental illness prior to their participation in the HOPE study. The most commonly described beliefs were that mental illness is caused by evil spirits and that patients with mental illness do not improve. ASHA workers also occasionally mentioned that they had believed mental illness patients were different from other patients and they did not know how to approach these patients.

On the other hand, some ASHA workers who had received training on mental health as part of the government programme were able to identify mental illness. Others had personal experiences with mental illness, which influenced their beliefs.

Yeah, mental illness ... When I was small my mom also got the same-the god rituals, all those things we did ... Once my father told that all those things are not required, and he will take her to NIMHANS [Psychiatric institution] and get [her] treated. [A111]

Most ASHAs said that following their participation in the HOPE study, they understood that mental illness is a treatable condition. ASHA workers identified sitting alone, talking to oneself and refusing to leave the house as symptoms of mental illness.

Some of the most commonly cited causes of mental illness were the patients' co-morbid physical problems and 'overthinking'.

Before, she [a patient] was thinking too much about her disorder and about the family it seems. Because she's staying with her husband, and he is too old now. And her children have shifted to Bangalore it seems, nobody is taking care of her now. So she was thinking too much about her life, how it will run and what should she do. All those things. And in the mean time she got this sugar [diabetes] also, how to treat, all those things she was thinking too much. [A116]

Other causes included family problems, family deaths, and other stressors. When asked how to treat mental illness, most ASHA workers said that controlling the patient's physical disorder would cure the mental illness. Every ASHA worker seemed to believe that mental illness is always temporary, and always treatable.

Ok, she [a patient] got into depression because she got diabetes. So when the diabetes is under control and it's treatable, when she got to know that, she became normal slowly. [A104]

Another common belief among ASHA workers was that mentally ill patients needed to stop worrying too much, in order to relieve tension.
Their understanding of the complex aetiology of CMD remained incomplete at the time of the interviews. No ASHA worker mentioned any genetic or neurological causes of mental illness. Additionally, they had a simplified understanding of CMD treatment, failing to mention any forms of psychotherapy, and their common advice to 'stop worrying' was met with resistance.

Patients are saying that we are telling them not to think too much about their problems ... but the patients are facing family problems and they haven't constructed their house. They have that work and this work and all those things, but still we are telling them not to think. They asked, how can they be without thinking? [A105]

Several ASHA workers mentioned a need for more formal and detailed training about mental illness, particularly to help them become more adept at giving advice about treatment for mental illness.

\section{Treatment facilitators}

Positive relationships between patients and ASHA workers helped in treatment compliance and retention, and helped improve patients' understanding of their health conditions. ASHA workers frequently told stories where patients gave them credit for their improvement.

Overall the patients told that even we [ASHA] are taking care of their health so much, why can't they take [responsibility for their own health]? Now, even they start taking care of their health, they thank me for my help and support. Before the patient was not knowing anything about her health condition and how to take care of health. But now she has learned everything and whenever I do home visit, she will be telling, thankful, that because of me only she got the treatment and learned many things. [A116]

ASHAs viewed the HLG sessions as very helpful for patients, especially due to the friendships that patients made in the groups. In addition, they believed participation in HLG sessions helped patients bring structure and regularity to their schedule.

Usually ... if a person is going [to the] office ... every day they will eat at 8:30. If the person is staying at home, they won't [eat at] this exact time-they'll eat whenever they are free. The same thing happens [to the patients] - if they are taking the sessions, they'll come regularly in time and start doing [the techniques]. If they are staying at home, it's not possible for them. [A107]

Many ASHA workers claimed to have implemented lifestyle changes in their own lives following participation in HOPE, and to have disseminated their new knowledge informally among other ASHA workers and village members.

Perhaps the most frequently mentioned facilitator to treatment was that it was offered free of cost. ASHA 
workers often used this to motivate patients to attend screening fairs and sessions. ASHA workers occasionally mentioned that support from patients' families helped facilitate treatment, with families pushing the patients to go to sessions and take care of their health.

The family members are cooperative with me, they [are the ones] sending the patient [to us]. The family members also need that-[they need] the mother and father to get treated and become well soon, so they were sending [them to us]. [A113]

\section{Treatment barriers}

An important barrier to treatment was the lack of accessibility to both the district hospitals and to the HLG sessions at the PHCs. Every ASHA worker mentioned at least one accessibility-related barrier during her interview, including distance to the PHCs, the transportation costs, the financial consequences of losing a full day's work, disability-related issues, and work and family obligations.

As described previously, negative relationships between patients and ASHA workers were occasionally a barrier to treatment. Also, ASHAs involvement in the governmentsponsored ASHA programme and/or their personal housework sometimes interfered with effective participation in the HOPE study.

ASHA workers occasionally mentioned that some patients were particularly distrustful of the medical system and government health programmes.

At the start when we were visiting village members, the villagers would think that we are coming for some survey, and we'll do our [own] work, we will not do anything for the villagers. The villagers were refusing to take me [ASHA] into the home ... Usually we wear a pink colored sari, so when we wear that and go [to houses], they [villagers] think that we are coming for some survey-larvae survey or some other thing, leprosy ... It's not going to benefit them. So they were not responding properly to us. [A117]

This lack of trust occasionally extended to HOPE study staff, especially regarding the study's intentions.

Some of the patients were saying that [study staff] will come 1 or 2 times and they won't take care [of us] then-they'll do whatever they want ... This means, that some NGOs will come for 1 or 2 days and the NGOs will give all [kinds of] hopes to the villagers, but they will leave [after 1 or 2 days] and they will not come back again. [Patients thought] that even the HOPE study staff also do same thing, that they'll come today and then the next day they won't come. [A107]

Similarly, ASHAs mentioned that some patients did not understand the purpose of medical procedures like blood tests, which scared them away from taking part in the HOPE study. With such patients, however, ASHA workers and study staff were able to intervene and explain the purpose of study procedures.

\section{ASHA perspectives on the HOPE study}

Most ASHA workers were able to remember the general procedures of the HOPE study and the content of the HLG sessions, without prompting. Some ASHAs were unable to recall all of the topics of the HLG sessions. One ASHA worker was unable to recall any of the components of the HOPE study.

In general, ASHA workers believed the HOPE study was useful for patients, claiming that the health of almost all participating patients improved. They reported that most patients enthusiastically participated in HLG sessions and practiced the tasks taught, and some patients still maintained the relationships they formed with other patients.

[One patient with] BP [blood pressure] and sugar [diabetes]. Before, his BP and sugar was not under control. Once he started practicing our techniques, it became under control, and now also he's practicing the things ... he's taking medication regularly, and walking every day. And maintaining the diet pattern, which was not there when he first came here. And he is not taking tension for every matter. He's well and good now. [A108]

The ASHA workers claimed that patients were grateful for the opportunity to participate in the HOPE study, especially because many patients had not known about their various illnesses, and the screening fairs helped them become aware.

... some patients were not at all checked for the BP and sugar [diabetes]. Once they got checked and got to know they have BP and sugar, the treatment got started. Those people were telling that because of me only they got to know about their health condition. [A113]

ASHA workers were reticent when asked about challenges associated with the HOPE study. The most consistent challenge they identified was irregular attendance. They occasionally told stories of patients whose health declined following the end of the study. ASHAs believed that these declines usually occurred because of a death in the patient's family and/or because patients stop practicing the tasks taught during the HLG sessions.

A: But one patient, his wife died recently, so because of that he's a bit ... sad. Before her death he was doing [the techniques] it seems. Now he is not doing anything.

SB: He's not doing anything ... Are there any techniques he's using at all?

A: No. He's in a sad [mood] so he's not ...

SB: How often do you visit him?

A: Every day I go. 
SB: You see him every day? Have you tried to remind him about the techniques?

A: Every day I'm telling but he's not ...

SB: Why doesn't he do it, what does he say?

A: He's not refusing and he's not doing. He's just listening. He's not interested in that now. [A105]

ASHA workers did not have many suggestions for improving the HOPE study. Most simply asked that the programme be expanded to include more patients and be of longer duration. Some requested that additional topics be added, like menstrual health and hygiene. Several ASHA workers asked for more information regarding mental illnesses other than CMD, more details on NCD and more formal training in mental health intervention.

\section{DISCUSSION}

Through this study, we obtained insight into the perspectives and beliefs of ASHA workers about their participation in the HOPE study, a community-based collaborative care mental health intervention. The ASHA workers expressed enthusiasm and satisfaction about their role and participation in HOPE. They perceived the HOPE study as helpful and necessary, and almost all of the ASHAs requested that the HOPE study continue and expand to additional districts. ASHA workers were also able to identify how multiple key intervention strategies, guided by our theoretical framework, improved the participants' self-efficacy, which in turn facilitated their access to treatment. Specific intervention components mentioned included the provision of social support as well as the many opportunities to learn problem solving skills, both through direct skills training and by observing other group members go through this process.

ASHAs consistently identified interpersonal interactions between patients and their families, other patients, and the ASHAs themselves as being key to the intervention's success. ASHAs also noted that the community screening fairs and group sessions were extremely helpful for participants-and that they seemed to become more motivated to implement the lifestyle changes taught during the HLGs once they learnt more about their disorders. Additionally, ASHAs believed that following the skills training, most participants felt confident in their ability to manage their health following HOPE, indicating increased selfefficacy, which is a central construct in SCT. ${ }^{28} 29$

An important theme that emerged was how the ASHA workers' beliefs about mental illness changed following their involvement in the HOPE study. Previous to HOPE, most ASHAs claimed to have held misconceptions and stigmatising beliefs about mental illness, including that mental illness was caused by evil spirits and could not be treated, and that patients with mental illness were different from others. Some ASHA workers had never heard of mental illness. This is consistent with the literature, which shows low mental health literacy among ASHAs. ${ }^{21} 232430$

Following their participation in HOPE, most ASHA workers were able to identify a consistent pattern of symptoms and treatments, and advocated for approaching patients with kindness and empathy, which is also consistent with existing literature. ${ }^{23}{ }^{30}$ Notably, the ASHAs had astute insights about comorbidity, often discussing the effects that physical disorders have on mental health. This is a unique feature of HOPE, which recruited patients with comorbid CMD and NCD. These results provide additional evidence that ASHA workers' beliefs and knowledge are receptive to change. ${ }^{24}{ }^{31}$ Nevertheless, ASHA workers are rarely targeted by community mental health awareness campaigns. ${ }^{32} 33$ Considering the importance of ASHA workers in rural healthcare and their increasing participation in mental health interventions, ${ }^{27}{ }^{34-36}$ they should be included in mental health education efforts.

Despite their significant gains in mental health knowledge, ASHA workers failed to understand the complex aetiology of mental illness, often identifying the comorbid medical condition as the only cause. Previous studies have also shown that ASHA workers rarely identified genetics, trauma or brain disease as causes of mental illness. ${ }^{24} 30$ Many ASHAs believed that CMD is always a transient condition, and that treating the comorbid medical condition and 'not worrying' were quick fixes. Additional training is clearly needed to empower ASHAs to provide mental health support to patients, perhaps similar to the Atmiyata intervention that trained community volunteers to provide basic mental illness counselling. ${ }^{33}$ Indeed, several ASHA workers asked for more training in mental health.

ASHA workers play a key role in connecting rural patients to healthcare systems, and findings from this study highlight the importance of this role. The interviews demonstrated that many ASHAs formed strong positive relationships with patients that encouraged the patients to attend sessions, practice techniques, and make concerted efforts to improve their health. ASHA workers helped recruit patients to the screening fairs and sessions by explaining the study aims, providing assurances and encouragement, and dispelling pervasive misconceptions about study procedures like blood draws. Additionally, most ASHAs expressed enthusiasm for helping patients, often citing rewarding experiences in which their patients thanked them or credited them for their efforts. This is also consistent with previous research, which shows that ASHAs are often motivated by community service, connection and feelings of responsibility towards patients. ${ }^{25}{ }^{26}$ The HOPE study is a novel attempt at extending the collaborative care model to the community to take advantage of the bidirectional interactions between patients and their environment specified in SCT, through skills-building, feedback, and provision of social support. ${ }^{28}{ }^{29}$ This process focused on behaviours likely to improve both mental and physical health, such as healthy eating, stress reduction, exercise, moderate drinking and 
tobacco use cessation. ASHA-patient relationships were likely a key factor in this community outreach by helping to foster trust and education on health issues.

There are several important limitations to consider when interpreting the results of this study. The realtime translation by an interpreter may have caused some nuance and complexity to be lost. Also, ASHA workers rarely discussed challenges they faced or suggestions for improvement. It is likely that, despite being assured that their honest opinions were welcome, some of the ASHAs were reluctant to criticise the study and were telling researchers what they thought they wanted to hear. Finally, some ASHAs needed to be reminded of HLG topics or study procedures before answering questions, making it difficult to get accurate perspectives. This speaks to a need for reminder sessions after the intervention ends to ensure knowledge retention and long-term sustainability of the intervention.

Despite these limitations, this qualitative study is the first study to explore the perspectives of ASHA workers about their participation in a community-based mental health intervention, and the results have several implications for public health policy and practice. Multiple barriers exist to accessing adequate treatment in rural areas, including financial concerns, transportation difficulties, low health literacy and a lack of trust of government health systems including the PHCs. These findings have been identified by other studies. ${ }^{11} 33$ ASHA workers may help mitigate these issues, as shown through their experience with HOPE, and ASHAs have previously acted as friends and advocates for vulnerable patients. ${ }^{20}$ Additionally, as members of the communities that they serve, ASHAs may help sustain long-term effects of community-based mental health intervention such as the HOPE study-as they continue to check in frequently with their patients and spread knowledge with others in their village. Given the positive effects of ASHA involvement, and their enthusiasm for participating in the intervention, they should be part of community-based interventions of NCD and CMD in rural India. Future community mental health interventions should educate and empower community health workers to most effectively help rural patients.

\begin{abstract}
Acknowledgements The authors thank Ms Aishwarya, who translated all the interviews with ASHA workers, and Mr Noorulla, who provided important logistical support. We are also grateful to the study manager Mr Anil Lobo and other members of the HOPE study staff, for recruiting ASHA participants and organising logistics. US project coordinators, Ms Amanda Mazur and Ms Hadley Burroughs, helped with mentorship and training and Ms Mersal Samini came up with an earlier version of this study. Faculty and staff at SJRI and UC Berkeley, including Ms Isabelle Charles and Dr Ryan Fernandes, provided additional mentorship and support to the first author.
\end{abstract}

Contributors SB designed the study, acquired and analysed all data, and drafted the manuscript. KS and ME supervised data acquisition and analysis, and drafted and revised the manuscript. Guarantor: SB.

Funding This work was primarily supported by the MHIRT (Minority Health International Research Training) Fellowship disbursed from the NIH (National Institutes of Health) to the University of California, Berkeley. It was additionally supported by NIH grant number R01MH100311 (Ekstrand and Srinivasan, MPI), which funded the parent study, 'Improving Mental Health through Integration with Primary Care in Rural Karnataka' (IRB 12-10470).
Competing interests None declared.

Patient consent for publication Not applicable.

Ethics approval The study was approved by the Institutional Review Boards (IRB) of the University of California, San Francisco and Berkeley (IRB 18-24388, reference \#249422), and by the Institutional Ethics Committee (IEC) of St. John's Medical College and Hospital (reference \#142/2018). Informed consent from every ASHA worker who participated was obtained prior to study procedures-ASHAs were given a study information sheet and consent form, both in Kannada, and any questions they had were answered.

Provenance and peer review Not commissioned; externally peer reviewed.

Data availability statement Data are available upon reasonable request. The datasets generated and analyzed during the current study are not publicly available due to confidentiality and anonymity concerns for individual ASHA participants.

Supplemental material This content has been supplied by the author(s). It has not been vetted by BMJ Publishing Group Limited (BMJ) and may not have been peer-reviewed. Any opinions or recommendations discussed are solely those of the author(s) and are not endorsed by BMJ. BMJ disclaims all liability and responsibility arising from any reliance placed on the content. Where the content includes any translated material, BMJ does not warrant the accuracy and reliability of the translations (including but not limited to local regulations, clinical guidelines, terminology, drug names and drug dosages), and is not responsible for any error and/or omissions arising from translation and adaptation or otherwise.

Open access This is an open access article distributed in accordance with the Creative Commons Attribution Non Commercial (CC BY-NC 4.0) license, which permits others to distribute, remix, adapt, build upon this work non-commercially, and license their derivative works on different terms, provided the original work is properly cited, appropriate credit is given, any changes made indicated, and the use is non-commercial. See: http://creativecommons.org/licenses/by-nc/4.0/.

ORCID iD

Stuti Bansal http://orcid.org/0000-0003-2375-5417

\section{REFERENCES}

1 Institute for Health Metrics and Evaluation (IHME). Findings from the global burden of disease study 2017. Seattle, WA: IHME, 2018.

2 Whiteford HA, Degenhardt L, Rehm J, et al. Global burden of disease attributable to mental and substance use disorders: findings from the global burden of disease study 2010. The Lancet 2013;382:1575-86.

3 Arvind BA, Gururaj G, Loganathan S, et al. Prevalence and socioeconomic impact of depressive disorders in India: multisite population-based cross-sectional study. BMJ Open 2019;9:e027250.

4 Charlson FJ, Baxter AJ, Cheng HG, et al. The burden of mental, neurological, and substance use disorders in China and India: a systematic analysis of community representative epidemiological studies. Lancet 2016;388:376-89.

5 Felker B, Yazel JJ, Short D. Mortality and medical comorbidity among psychiatric patients: a review. Psychiatr Serv 1996;47:135663. Dec.

6 Ross CA, Goldner EM. Stigma, negative attitudes and discrimination towards mental illness within the nursing profession: a review of the literature. J Psychiatr Ment Health Nurs 2009;16:558-67.

7 Math SB, Chandrashekar CR, Bhugra D. Psychiatric epidemiology in India. Indian Journal of Medical Research 2007;126:183.

8 World Health Organization. Mental health atlas 2017. Geneva, 2018.

9 India State-Level Disease Burden Initiative Mental Disorders Collaborators. The burden of mental disorders across the states of India: the global burden of disease study 1990-2017. Lancet Psychiatry 2020;7:148-61.

10 Jacob KS, Sharan P, Mirza I, et al. Mental health systems in countries: where are we now? Lancet 2007;370:1061-77.

11 Kumar A. Mental health services in rural India: challenges and prospects. Health 2011;3:757-61.

12 INDIA P. Census of India 2011 provisional population Totals.

13 National Rural Health Mission (India). National Rural Health Mission: Meeting People's Health Needs in Rural Areas: Framework for Implementation, 2005-2012. National Rural Health Mission, Ministry of Health \& Family Welfare, Government of India, 2007.

14 Scott K, George AS, Ved RR. Taking stock of 10 years of published research on the ASHA programme: examining India's national community health worker programme from a health systems perspective. Health Res Policy Syst 2019;17:29. 
15 Sundararaman T, Ved R, Gupta G, et al. Determinants of functionality and effectiveness of community health workers: results from evaluation of ASHA program in eight Indian states. BMC Proc 2012;6.

16 Nyamathi AM, Shin SS, Sinha S, et al. Sustained effect of a community-based behavioral and nutrition intervention on HIV-related outcomes among women living with HIV in rural India: a quasi-experimental trial. J Acquir Immune Defic Syndr 2019;81:429-38.

17 Nyamathi A, Hanson AY, Salem BE, et al. Impact of a rural village women (Asha) intervention on adherence to antiretroviral therapy in southern India. Nurs Res 2012;61:353-62.

18 Nyamathi A, Salem BE, Meyer V, et al. Impact of an Asha intervention on depressive symptoms among rural women living with AIDS in India: comparison of the Asha-Life and usual care program. AIDS Educ Prev 2012;24:280-93.

19 Nyamathi A, Ekstrand M, Salem BE, et al. Impact of Asha intervention on stigma among rural Indian women with AIDS. West $J$ Nurs Res 2013;35:867-83.

20 Srivastava N, Nyamathi AM, Sinha S, et al. Women living with AIDS in rural southern India: perspectives on mental health and lay health care worker support. J HIV AIDS Soc Serv 2017;16:170-94.

21 Armstrong G, Kermode M, Raja S, et al. A mental health training program for community health workers in India: impact on knowledge and attitudes. Int J Ment Health Syst 2011;5:17.

22 Menon J, Joseph J, Thachil A, et al. Surveillance of noncommunicable diseases by community health workers in Kerala: the epidemiology of noncommunicable diseases in rural areas (ENDIRA) study. Glob Heart 2014;9:409-17.

23 Salve HR, Babu S. Attitude about mental illness of health care providers and community leaders in rural Haryana, North India. Indian Journal of Community Health 2014;26:374-8.

24 Almanzar S, Shah N, Vithalani S, et al. Knowledge of and attitudes toward clinical depression among health providers in Gujarat, India. Ann Glob Health 2014;80:89-95.

25 Gopalan SS, Mohanty S, Das A. Assessing community health workers' performance motivation: a mixed-methods approach on India's accredited social health activists (ASHA) programme. BMJ Open 2012;2. doi:10.1136/bmjopen-2012-001557. [Epub ahead of print: 2709 2012]
26 Wahid SS, Munar W, Das S, et al. 'Our village is dependent on us. That's why we can't leave our work'. Characterizing mechanisms of motivation to perform among Accredited Social Health Activists (ASHA) in Bihar. Health Policy Plan 2020;35:58-66.

27 Srinivasan K, Mazur A, Mony PK, et al. Improving mental health through integration with primary care in rural Karnataka: study protocol of a cluster randomized control trial. BMC Fam Pract 2018;19:1-2.

28 Bandura A. Social cognitive theory and exercise of control over HIV infection. In: DiClemente RJ, Peterson JL, eds. Preventing AIDS: theories and methods of behavioral interventions. New York: Plenum, 1994: 25-59.

29 Bandura A. Social learning theory. Englewood Cliffs, NJ: PrenticeHall, 1977.

30 Shah QN, Dave PA, Loh DA, et al. Knowledge of and attitudes towards mental illness among ASHA and Anganwadi workers in Vadodara district, Gujarat state, India. Psychiatr Q 2019;90:303-9.

31 Hofmann-Broussard C, Armstrong G, Boschen MJ, et al. A mental health training program for community health workers in India: impact on recognition of mental disorders, stigmatizing attitudes and confidence. Int J Cult Ment Health 2017;10:62-74.

32 Maulik PK, Devarapalli S, Kallakuri S, et al. Evaluation of an antistigma campaign related to common mental disorders in rural India: a mixed methods approach. Psychol Med 2017;47:565-75.

33 Shields-Zeeman L, Pathare S, Walters BH, et al. Promoting wellbeing and improving access to mental health care through community champions in rural India: the Atmiyata intervention approach. Int $J$ Ment Health Syst 2017;11:6.

34 Chatterjee S, Naik S, John S, et al. Effectiveness of a communitybased intervention for people with schizophrenia and their caregivers in India (COPSI): a randomised controlled trial. Lancet 2014;383:1385-94.

35 Maulik PK, Kallakuri S, Devarapalli S, et al. Increasing use of mental health services in remote areas using mobile technology: a pre-post evaluation of the smart mental health project in rural India. J Glob Health 2017;7:010408.

36 Patel V, Weiss HA, Chowdhary N. The effectiveness of a lay health worker led intervention for depressive and anxiety disorders in primary care: the MANAS cluster randomized trial in Goa, India. Lancet 2010;376:2086. 